\title{
STAT3 Inhibitor TTI-101
}

National Cancer Institute

\section{Source}

National Cancer Institute. STAT3 Inhibitor TTI-101. NCI Thesaurus. Code C151932.

An orally bioavailable, binaphthol-sulfonamide-based inhibitor of signal transducer and activator of transcription 3 (STAT3), with potential antineo plastic activity. Upon oral administration, the STAT 3 inhibitor TTI-101 specifically targ ets and binds to the phosphotyrosyl peptide binding site within the Src homology 2 (SH2) domain of STAT3. This inhibits the Janus kinase (JAK)-mediated tyrosine phosphorylation and activation of STAT 3. This impedes nuclear translocation of STAT3, prevents STAT3 binding to responsive gene promoters and blocks STAT3-mediated regulation of gene expression. STAT 3 regulates the transcription of genes involved in several cellular functions. STAT 3 is constitutively activated in a variety of human cancers and plays a key role in neoplastic transformation, uncontrolled tumor cell proliferation, resistance to apoptosis, metastasis, immune evasion, tumor ang iogenesis, epithelial-mesenchymal transition (EMT) and the Warburg effect. 